\title{
The Maximum Standardized Fdg Uptake on Pet-Ct in Patients with Small Cell Lung Cancer
}

\author{
Huynh Quang Huy* \\ Radiology Department, Vietnam
}

Received: 䀺: November 14, 2018; Published: 眥: November 26, 2018

*Corresponding author: Huynh Quang Huy, Radiology Department, Vietnam, 2 Quang Trung street, District 10, Ho Chi Minh city, Vietnam

\begin{abstract}
Background: Small-cell lung cancer (SCLC) accounts for 15\%-20\% of all lung cancer cases. PET-CT has become increasingly used as an initial staging tool in patients with SCLC. PET has emerged in the last decade as an important tool in the staging and delineation of disease for conformal radiotherapy planning of non-SCLC, but not yet been widely incorporated into the staging of SCLC.
\end{abstract}

Objective: The objective of the present study is to assess whether the maximum SUV (SUVmax) in PET-CT correlates with tumor size, lymph node metastasis, distant metastasis in patients with SCLC.

Methods: Patients with SCLC who underwent 18F-FDG PET-CT scans before the treatment were included in the study at Bach Mai hospital of Vietnam, from November 2015 to May 2018. The primary tumor and secondary lesion SUVmax was calculated; the tumor size was measured; the T-N-M status was determined mainly by FDG PET-CT imaging according to the 8th Edition of the TNM Classification for Lung Cancer were recorded. An evaluation was made of the linear relationship between tumor size, $\mathrm{T}$ stage, $\mathrm{N}$ stage, and $\mathrm{M}$ stages of the patients and their SUVmax using Spearman's correlation.

Results: 37 cases (34 men and 3 women; age range 38 - 81 years, median 64 years) were analyzed. The average of primary tumor size and SUVmax were $5.95 \pm 2.77 \mathrm{~cm}$ and $10.21 \pm 4.75$, respectively. The SUVmax of primary tumor is significantly greater than that of nodal and distant organ metastasis $(10.21 \pm 4.75$ vs $8.20 \pm 4.35$ and $6.44 \pm 3.17, p<0,01)$. There was a moderate correlation between SUVmax and tumor size ( $r=0.596$, $\mathrm{p}<0.001)$, tumor stage $(\mathrm{r}=0.502, \mathrm{p}<0.01)$ but not significant with nodal stage $(\mathrm{r}=-0.218, \mathrm{p}=0.194)$, metastasis stage $(\mathrm{r}=-0.055, \mathrm{p}=0.747)$, and overall stage $(r=-0.060, p=0.725)$.

Conclusion: SUVmax was significantly correlated with tumor size, but not with distant metastases or lymph node involvement. Therefore, SUVmax on positron emission tomography is not predictive of the presence of metastases in patients with SCLC.

Keywords: Maximum Standardized Fdg Uptake (Suvmax); Primary Tumor; Lymp Node; Distant Metastases; Small Cell Lung Cancer (Sclc)

Abbreviations: SCLC: Small cell lung cancer; FDG: Fluorodeoxyglucose; PET: Positron Emission Tomography; KW: Kruskal-Wallis; SR: Spearman Rank

\section{Introduction}

Lung cancer is the leading cause of cancer death worldwide [1,2]. Small cell lung cancer (SCLC) represented approximately 10-15\% of all lung cancers $[3,4]$. Smoking is the main risk factor for SCLC, approximately $95 \%$ of these patients were smokers [5]. SCLC is characterized by the low degree of differentiation, shorter doubling time and high sensitivity to chemotherapy and radiotherapy. Each year, $13 \%$ of all newly diagnosed lung cancer patients are diagnosed with SCLC [6]. Approximately $39 \%$ of patients with SCLC are diagnosed with LS disease treated with chemotherapy and definitive radiation therapy [6]. Staging information is essential because of the high propensity for metastatic disease in SCLC, and the identification of metastases can spare patients from the toxicity associated with thoracic radiotherapy. Furthermore, in those patients who do receive radiotherapy, knowing the exact extent of intrathoracic disease may permit more accurate treatment volume delineation. PET has emerged in the last decade as an important tool in the staging and delineation of disease for conformal radiotherapy planning of non-SCLC. In 2009, Medicare approved the use of PET for the initial staging of SCLC. It is believed that PET may more accurately detect patients with extensive-stage disease than CT-staging alone. Fluorodeoxyglucose (FDG) positron emission tomography (PET) has been widely embraced as part of the routine workup for non-small-cell lung cancer (NSCLC). In 
contrast, FDG-PET has not yet been widely incorporated into the staging of SCLC [7]. The objective of the present study is to assess whether the maximum SUV (SUVmax) in PET-CT correlates with tumor size, lymph node metastasis, distant metastasis in patients with SCLC.

\section{Methods \\ Patient Selection}

The records of 37 patients newly diagnosed with SCLC between November 2015 and October 2018 at Bach Mai hospital were evaluated retrospectively. The subjects were examined by Fluorodeoxyglucose PET-CT and TNM stage of SCLC. A total of 34 males and 3 females were included in the study, with a mean age $62.0 \pm 9.4$ years (range 38-81 years). Patients were excluded for the following reasons: primary lesion smaller than $1 \mathrm{~cm}$ (to ensure feasibility of PVC), histology could not be confirmed or was confirmed as other than SCLC, type I diabetes, prior history of lung cancer or other prior cancer within the previous 5 years, previous therapy or surgical staging for SCLC before PET.

\section{FGD-PET Imaging}

All patients underwent diagnostic and/or staging FDG-PET-CT prior to biopsy or therapy. Patients were asked to fast at least $6 \mathrm{~h}$ before the FDG-PET-CT scan. All patients had a glucose level below $180 \mathrm{mg} / \mathrm{dl}$ and were injected intravenously with $0.15-0.20 \mathrm{mCi} /$ $\mathrm{kg}$ (7-12mCi) FDG. At 45-60 min after the injection, data were acquired from the vertex to the upper thigh. Immediately after CT, a PET scan (PET/CT Biograph True Point - Siemens, Germany) was performed for about $25 \mathrm{~min}$, with seven to eight bed positions and $3 \mathrm{~min} /$ position. PET images were reconstructed iteratively with CT data for attenuation correction, using an inline integrated Siemens Esoft Workstation system. Computerized tomography integrated positron emission tomography fusion images in transaxial, sagittal, and coronal planes were evaluated visually, and the SUVmax of lesions was obtained from transaxial images.

\section{CT Determination of Tumor Size}

Tumor size was determined by averaging all 3 diameters of the primary tumor, measured on the mediastinal windows of the chest CT, using printed films. CT scans were obtained either at our institution or by the referring physician.

\section{Statistical Analyze}

Nonparametric, rank-based statistical methods were chosen because none of our measurements (maxSUV, tumor size, TNM stage) could be assumed to have a normal distribution. Comparisons were therefore performed using a Kruskal-Wallis (KW) nonparametric test. Correlations between pairs of variables (i.e., maxSUV versus tumor size) were evaluated using the Spearman rank (SR) correlation test. All analyses were conducted using SPSS version 22.0.

\section{Results}

The characteristics and SUVmax of the 37 SCLC cases are summarized in Table 1. When the cases were divided into three groups based on tumor size (group $1,<3 \mathrm{~cm}$; group 2, $>3 \mathrm{~cm}$ and $<5 \mathrm{~cm}$; and group $3,>5 \mathrm{~cm}$ ), tumor SUVmax was differ significantly between groups 1,2 and $3(\mathrm{p}=0.006)$. Considering all cases, tumor SUVmax was not significantly correlated with age, gender or TNM overall stage. The average of primary tumor size and SUVmax were $5.95 \pm 2.77 \mathrm{~cm}$ and $10.21 \pm 4.75$, respectively. The SUVmax of primary tumor is significantly greater than that of nodal and distant organ metastasis $(10.21 \pm 4.75$ vs $8.20 \pm 4.35$ and $6.44 \pm 3.17, \mathrm{p}<0.01)$ showed in the Figure 1. There was a moderate correlation between SUVmax and tumor size $(\mathrm{r}=0.596, \mathrm{p}<0.001)$, tumor stage $(\mathrm{r}=0.502$, $\mathrm{p}<0.01$ ) (Figure 2) but not significant with nodal stage $(\mathrm{r}=-0.218$, $\mathrm{p}=0.194)$, metastasis stage $(\mathrm{r}=-0.055, \mathrm{p}=0.747)$, and overall stage $(\mathrm{r}=-0.060, \mathrm{p}=0.725)$. Figure 3 shows the PET-CT images of patients with primary tumor, lung metastasis, mediastium node and brain metastasis.

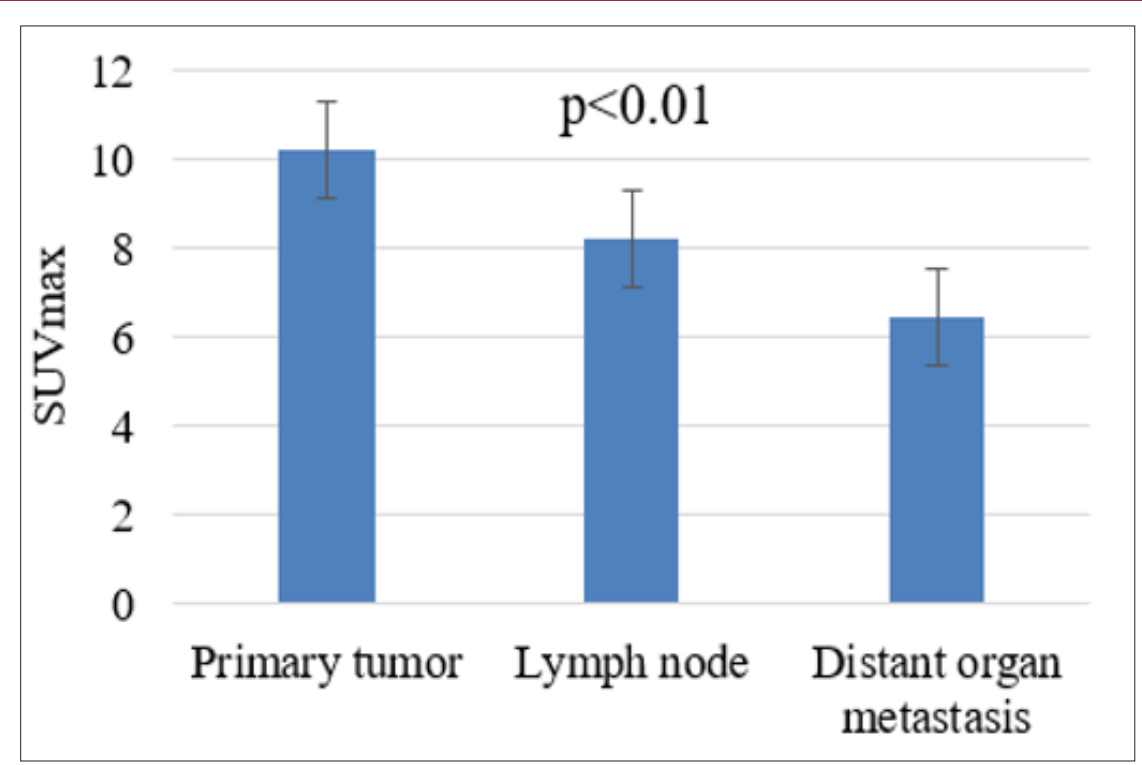

Figure 1: Comparison of SUVmax between primary tumors, lymph nodes and distant organ metastasis. 

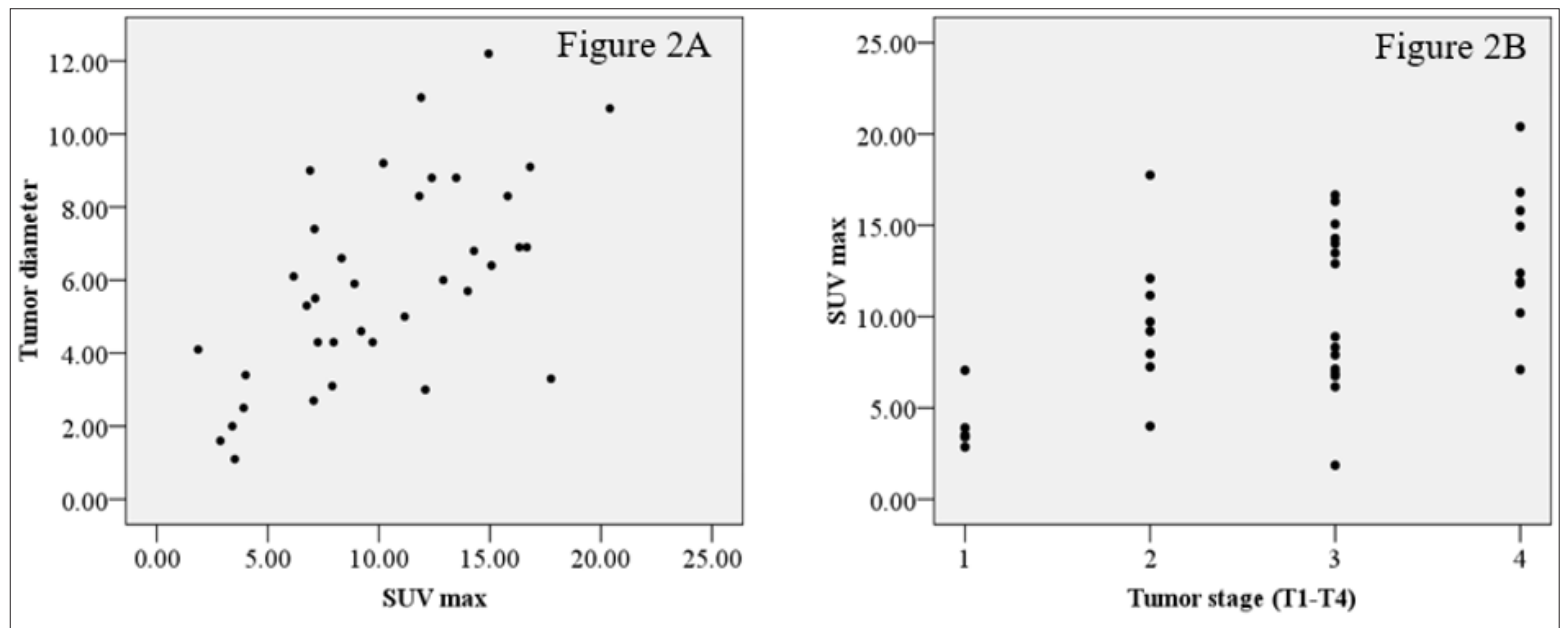

Figure 2: There is a significant correlation between SUVmax and tumor size (2A), tumor stage (2B).
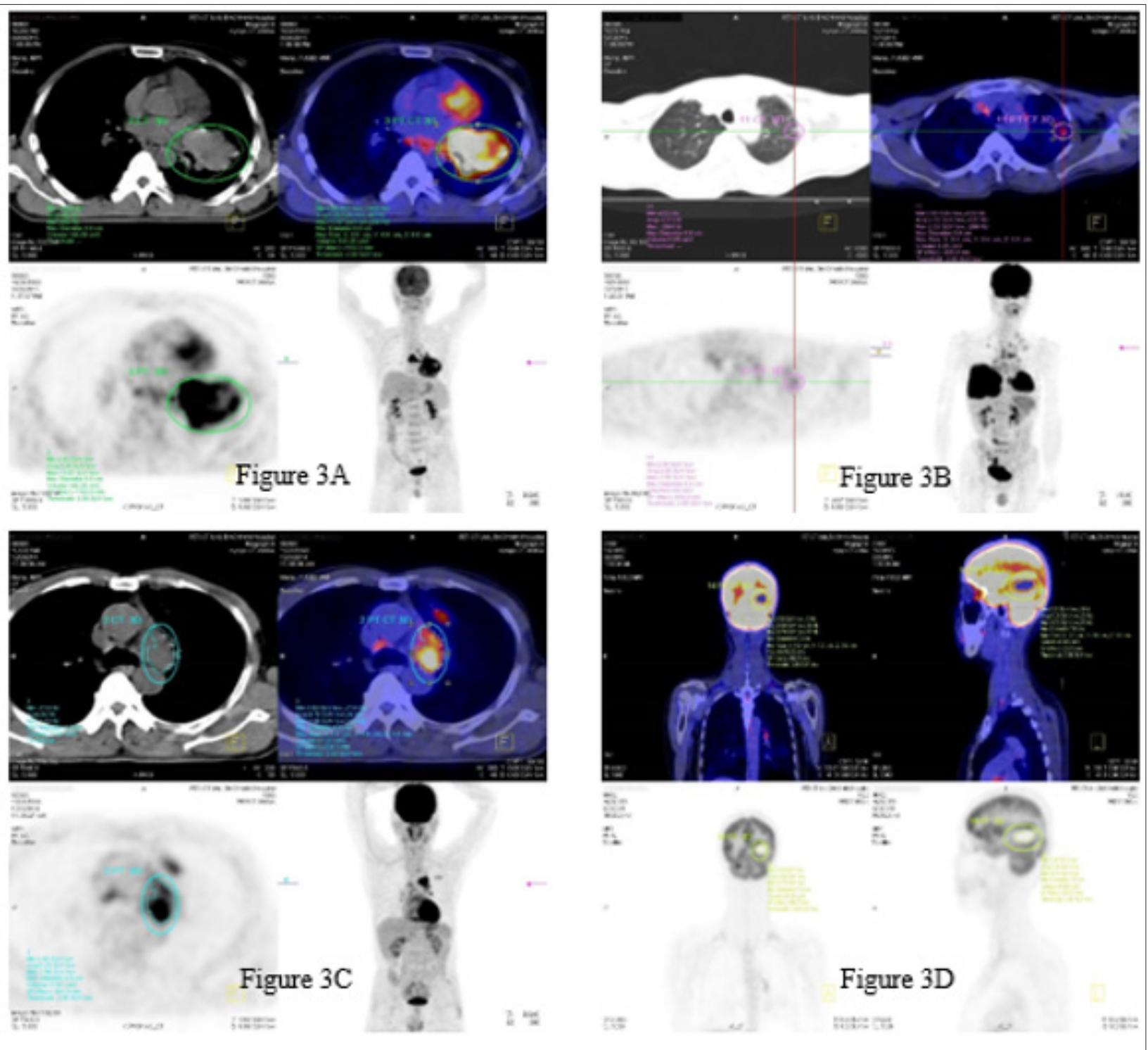

Figure 3: PET-CT images of patients with primary tumor (3A, diameter: 8.8cm, SUVmax: 13.97), lung metastasis (3B, diameter: $0.6 \mathrm{~cm}$, SUVmax: 2.55$)$, mediastinum node $(3 \mathrm{C}$, diameter: $4.9 \mathrm{~cm}$, SUVmax: 7.96$)$ and brain metastasis $(3 \mathrm{D}$, diameter: $7.0 \mathrm{~cm}$, SUVmax: 9.7). 
Table 1: Characteristics and SUVmax of the SCLC cases n (\%) SUV (mean \pm SD).

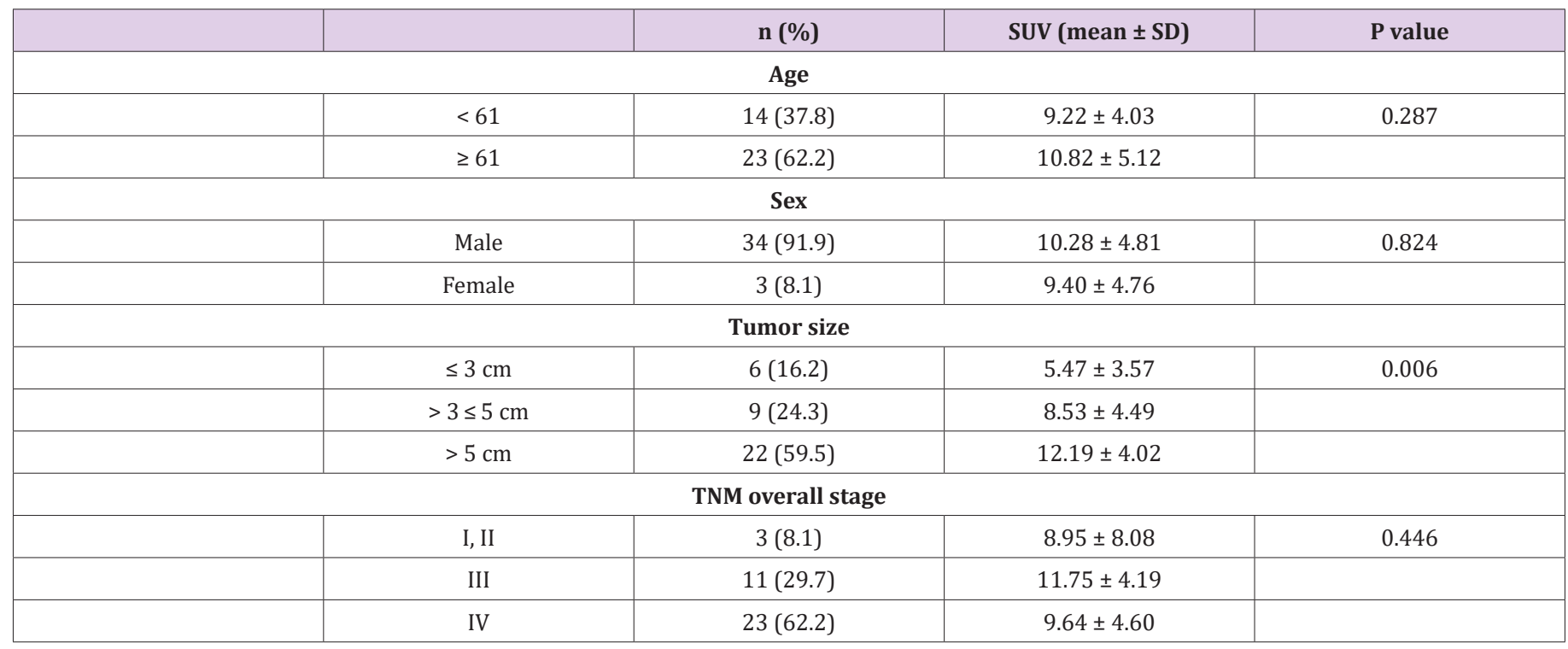

\section{Discussion}

Although CT or magnetic resonance imaging provides precise anatomical and morphological information, the role of FDG-PETCT has increased for diagnosis and staging of lung cancer. Recently, FDG uptake has been reported to be a prognostic factor in patients with lung cancer [5]. Patz et al. [8] demonstrated that patients with positive FDG-PET-CT results in treated lung cancer had a significantly worse prognosis than patients with negative results. Therefore, we examined whether SUVmax correlates with tumor size, TNM stage in patients with SCLC. Tumor size, tumor stage but not lymph node or distant metastases, was related to the tumor SUVmax. Doom et al. [9] also reported a strong significant association between tumor size and SUVmax in patients with NSCLC. Another study in patients with stage I NSCLC showed a significant association between the primary tumor, SUVmax and tumor size, with tumors $<3 \mathrm{~cm}$ having a significantly lower SUV than tumors $>3 \mathrm{~cm}$ [10]. Many studies regarding the correlation between SUVmax and other features such as histology, clinics in patients with NSCLC but no reports in SCLC has been found so far.

Fluorodeoxyglucose-PET-CT is already an indispensable modality for evaluating lymph node and distant metastases. Many reports have suggested that FDG-PET-CT is superior to CT in the accuracy of $\mathrm{N}$ - staging for lung cancer. Therefore, FDG-PET-CT is now regarded as the most accurate imaging modality for $\mathrm{N}$ - staging of lung cancer. However, a significant number of false-negative and false-positive findings of lung cancer, including $\mathrm{N}$-staging, on FDGPET-CT have been reported. Nambu et al. [11] demonstrated that the likelihood of lymph node metastasis increased with an increase in SUVmax of the primary tumor; for primary lung cancer with a SUVmax greater than 12 , the probability of lymph node metastasis was high, reaching $70 \%$, irrespective of the degree of FDG accumulation in the lymph node stations. They concluded that this finding would allow a more sensitive prediction of the presence of lymph node metastases, including the microscopic ones that cannot be detected by direct evaluation of lymph node stations.
Consistent with these results, Higashi et al. [12] documented in a multicenter study that the incidence of lymphatic vessel invasion and lymph node metastasis in NSCLC were associated with 18 F-FDG uptake, concluding that 18 F-FDG uptake by a primary tumor is a strong predictor of lymphatic vessel invasion and lymph node metastasis. In the present study, although tumor SUVmax was higher in patients with lymph node metastasis than in those without, the difference did not reach statistical significance. Ozgul et al observed that the frequency of lymph node metastasis was higher in adenocarcinomas (80.2\%) than in squamous cell carcinomas (71.4\%), suggesting that pathological subtype may be a significant factor associated with lymph node metastasis. In contrast, a previous study showed no difference in the frequency of lymph node metastasis between the two pathological subtypes [10].

Based on univariate analysis, Jeong et al. [13] concluded that metastasis detected by PET imaging, which can affect staging by aiding in the discovery of metastasis to contralateral lymph nodes or distant organs, was an insignificant factor, and that metastatic findings on PET had weak discriminative power. According to Cerfolio et al. [14], FDG-PET-CT does not replace the need for tissue biopsies for staging N1 or N2 lymph nodes, or metastatic lesions, as false positives and false negatives were observed in all stations in their study. However, FDG-PET-CT resulted in better patient selection before pulmonary resection. FDG-PET can also help in targeting areas for biopsy and identifying unsuspected N2 and Ml disease. In the present study, tumor SUVmax was not significantly correlated with distant metastases. This may be attributable to the finding of increased 18 F-FDG uptake by subclinical inflammatory lesions as well as by malignant tumors.

\section{Conclusion}

SUVmax was associated with tumor size, tumor stage but not with distant metastases or lymph node involvement. Thus, SUVmax determined by FDG-PET-CT is not predictive of the 
presence of metastases in patients with SCLC. Larger prospective and randomized analyses may potentially reveal more significant relationships.

\section{References}

1. Boloker G, Wang C, Zhang J (2018) Updated statistics of lung and bronchus cancer in United States. J Thorac Dis 10(3): 1158-1161.

2. Siegel RL, Miller KD, Jemal A (2018) Cancer statistics. CA Cancer J Clin 68(1): 7-30.

3. Byers LA, Rudin CM, (2015) Small cell lung cancer: where do we go from here? Cancer 121(5): 664-672.

4. Van Meerbeeck JP, Fennell DA, De Ruysscher DK (2011) Small-cell lung cancer. The Lancet 378(9804): 1741-1755.

5. Pesch B, Kendzia B, Gustavsson P, Jöckel KH, Johnen G, et al. (2012) Cigarette smoking and lung cancer-relative risk estimates for the major histological types from a pooled analysis of case-control studies. Int J Cancer 131(5): 1210-1219.

6. Govindan R, Page N, Morgensztern D, Read W, Tierney R, et al. (2006) Changing epidemiology of small-cell lung cancer in the United States over the last 30 years: analysis of the surveillance, epidemiologic, and end results database. J Clin Oncol 24(28): 4539-4544.

7. Ruben JD, Ball DL, (2012) The efficacy of PET staging for small-cell lung cancer: a systematic review and cost analysis in the Australian setting. J Thorac Oncol 7(6): 1015-1020.

\section{ISSN: 2574-1241}

DOI: $10.26717 / B J S T R .2018 .11 .002079$

Huynh QH. Biomed J Sci \& Tech Res

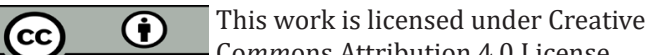
Commons Attribution 4.0 License

Submission Link: https://biomedres.us/submit-manuscript.php
8. Patz EF, Connolly J, Herndon J (2000) Prognostic value of thoracic FDG PET imaging after treatment for non-small cell lung cancer. AJR Am J Roentgenol 174(3): 769-774.

9. Dooms C, Van Baardwijk A, Verbeken E, Van Suylen RJ, Stroobants S, et al. (2009) Association between 18F-fluoro-2-deoxy-D-glucose uptake values and tumor vitality: prognostic value of positron emission tomography in early-stage non-small cell lung cancer. J Thorac Oncol 4(7): 822-828.

10. Ozgul MA, Gamze Kirkil, Ekrem Cengiz Seyhan, Erdoğan Çetinkaya, Güler Özgül, et al. (2013) The maximum standardized FDG uptake on PET-CT in patients with non-small cell lung cancer. Multidiscip Respir Med 8(1): 69.

11. Nambu A (2009) Relationship between maximum standardized uptake value (SUVmax) of lung cancer and lymph node metastasis on FDG-PET. Ann Nucl Med 23(3): 269-275.

12. Higashi K, Ito K, Hiramatsu Y, Ishikawa T, Sakuma T, et al. (2005) 18F-FDG uptake by primary tumor as a predictor of intratumoral lymphatic vessel invasion and lymph node involvement in non-small cell lung cancer: analysis of a multicenter study. J Nucl Med 46(2): 267-273.

13. Jeong HJ (2002) Determination of the prognostic value of [(18)F] fluorodeoxyglucose uptake by using positron emission tomography in patients with non-small cell lung cancer. Nucl Med Commun 23(9): 865870 .

14. Cerfolio RJ Bryant AS, Ohja B, Bartolucci AA (2005) The maximum standardized uptake values on positron emission tomography of a nonsmall cell lung cancer predict stage, recurrence, and survival. J Thorac Cardiovasc Surg 130(1): 151-159.

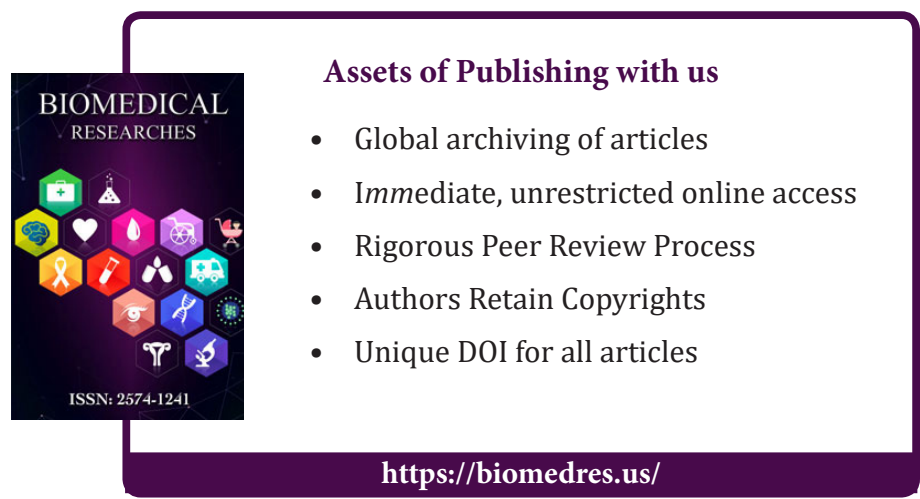

